\title{
Aplicações dos sistemas de informação: Quais as áreas de aplicação?
}

\section{Information systems applications: What are the application areas?}

\author{
Bertil P. Marques ${ }^{1,2}$ \\ bpm@isep.ipp.pt \\ ${ }^{1}$ Instituto Superior de Engenharia do Porto, Politécnico do Porto, Rua Dr. António Bernardino de Almeida, \\ 431, 4249-015 Porto, Portugal \\ ${ }^{2}$ GILT - Games, Interaction and Learning Technologies, ISEP, 4249-015 Porto, Portugal
}

DOI: 10.17013/risti.40.o

\section{Introdução}

Um Sistema de Informação (SI) é um sistema constituído por pessoas, procedimentos e equipamentos que recolhem, processam, armazenam, e distribuem informação com objetivos específicos. Tal como qualquer outro Sistema, um SI é composto por inputs (dados, instruções) e outputs (relatórios, cálculos). O SI processa os inputs e produz outputs que são disponibilizados ao utilizador final ou a outros sistemas. Também é normal ser incluído um mecanismo de feedback que faz o controlo da operação. Tal como qualquer outro Sistema, um SI opera num determinado ambiente (Turban et al., 1999). As organizações apresentam, na sua essência, um vasto conjunto de processos de gestão de informação. "qualquer organização (social), seja de que tipo for, pode e deve ser interpretada como um sistema de informação” (Rivas, 1989).

As principais funções do departamento de sistemas de informação (DSI), reflete com:

- Gestão de desenvolvimento de sistemas e gestão de projetos de sistemas;

- Planeamento, desenvolvimento e gestão da infraestrutura (hardware, software e comunicações):

- Gestão de operações de computadores incluindo o centro de informática;

- Recrutamento, formação e desenvolvimento de aptidões em SI;

- Proporcionar suporte técnico aos utilizadores finais. 
Atualmente, em muitas organizações, o DSI deixou de desempenhar um papel puramente de suporte técnico, tendo um papel também na gestão e na estratégia da organização.

- O diretor do DSI tende a deixar de ser um gestor técnico, de tecnologias, para passar a ser um gestor de informação (CIO - Chief Information Officer). (Marcondes, 2019)

As áreas de aplicação dos sistemas de informação não têm limite, uma vez que a sua aplicação, como já foi referenciado mais atrás não é um software, mas sim um sistema composto por inputs e por outputs (Turban et al., 1999).

Temos sistemas de informação com aplicações em áreas como as que poderemos identificar a seguir:

Sistema de Bibliotecas - Para a realização de projetos de sistemas de informação bem elaborados, o aspeto social e a cultura organizacional, são muito relevantes. O estudo de caso do Sistema de Bibliotecas da Unicamp trouxe para reflexão o quanto a Gestão do Conhecimento com abordagem em pessoas, pôde colaborar na criação de um ambiente favorável que proporcionou tanto a troca de conhecimento, como a condução do projeto. Os grupos Gestor e de Trabalho foram decisivos para o sucesso do empreendimento, reconhecendo a necessidade de se fomentar valores apropriados à inovação e ao compartilhamento do conhecimento, o estabelecimento de contatos pessoais, a análise de diferentes perspetivas, a abertura para a efetiva comunicação e o desenvolvimento de habilidades pessoais e profissionais (Andrade et al., 2019).

Educação - Ao ser aplicado numa escola privada, através dos estudos feitos foi encontrado o modelo de gestão da tecnologia da informação mais adequado para ser implantado na organização, o ITIL. Seria exigido que as empresas de TI se adequassem ao modelo de gestão, que estudasse minuciosamente sua metodologia, suas boas práticas e colocasse em prática em todos os processos que eram realizados dentro da escola (Almeida e Araújo, 2019).

Empresas de grandes dimensões (Cadeias Globais) - o verdadeiro desafio para as empresas não é simplesmente ter uma tecnologia e/ou sistema de informação sofisticado, mas sim descobrir como utilizar esses recursos para permitir a criação e troca de conhecimento entre pessoas nas organizações, tornando-se primordial para o sucesso das organizações. Atualmente, a maioria das empresas possuem setores de TI/SI, mas a diferença real vem de como elas foram utilizadas para aumentar sua competitividade organizacional e para sobreviver e prosperar num mundo e mercado industrial baseado em economia do conhecimento. Duas áreas importantes aceleraram o surgimento de sistemas modernos de gestão de conhecimento: tecnologias de rede e bases de dados locais. O aumento dos computadores em rede permitiu codificar, armazenar e compartilhar certos tipos de conhecimento de forma mais fácil e econômica do que nunca (Civi, 2000).

Saúde - Segundo a HISMM -Healthcare Information and Management Systems Society - eHealth é uma aplicação de Internet, utilizada em conjunto com outras tecnologias da informação, focada na melhoria de acesso, de eficiência, da efetividade e da qualidade dos processos clínicos e assistenciais necessários a toda a cadeia de prestação de serviços de saúde. O único objetivo é prover melhores condições de tratamento ao 
paciente e melhores condições de custeio ao Sistema de Saúde (Hummel, 2006). O Prontuário Eletrônico do Paciente (PEP) e Sistemas de Informação Hospitalar (SIH) são alguns exemplos de ferramentas de eHealth, que estão sendo utilizadas para integrar e compartilhar dados relacionados à saúde. O Prontuário Eletrónico do Paciente - PEP (ou EMR, do inglês Electronic Medical Records) é um documento digital e padronizado, constituído de um conjunto de informações históricas, acerca da saúde do paciente e da assistência a ele prestada em um determinado consultório ou qualquer estabelecimento de saúde. A construção do PEP baseia-se no princípio básico da integração da informação, que é coletada e registada em algum formato para que possa ser armazenada (Araújo et al., 2019).

Poderíamos continuar com exemplo, na área da engenharia civil, geotecnia e minas. $\mathrm{Na}$ área da educação ramificando desde a matemática à robótica. Na saúde, indo para o campo da assistência social e podendo também passar pela agricultura.

Assim, tem havido uma grande e constante evolução das aplicações dos sistemas de informação em diversas áreas, conduzindo à necessidade de mudanças nas organizações (principalmente expansões), e havendo sempre uma constante evolução das próprias tecnologias, leva-nos a encontrar nos 9 artigos desta edição da RISTI um fio condutor que são os sistemas de informação e em que áreas se aplicam. Como pudemos ver acima, conseguimos aplicar a todas as áreas se seguirmos o conceito definido por Turban et al. (1999). E também podemos nos 9 artigos desta edição fazer essa leitura.

Neste contexto este número da RISTI - Revista Ibérica de Sistemas e Tecnologias de Informação contém nove artigos, que foram selecionados depois de terem sido alvo de um exigente processo de avaliação pelos membros do conselho científico.

\section{Estrutura}

O primeiro artigo "Curadoria de chatbots: conceptualização, estratégias e indicadores de desempenho" da autoria Darío Reyes Reina e Clarice Cruz, tem o objetivo de analisar o fenómeno da curadoria de chatbots, para o qual foi realizado um estudo semiestruturado a profissionais experientes no desenvolvimento e na curadoria de chatbots. Este tema devido ao surgimento e expansão de inovações tecnológicas, (os chatbots,) tem ocasionado a formação de novas áreas de desempenho e/ou novos perfis profissionais. As principais descobertas do estudo, discutem-se sobre a relevância da curadoria de chatbots e possíveis compartilha das linhas de pesquisa.

O segundo artigo "Utilización de la Teoría de la Información para evaluar el comportamiento de la estabilidad estática en amputaciones transtibiales" da autoria de Lely A. Luengas-C., Daissy C. Toloza C. e Luis F. Wanumen, a teoria da informação (TI) foi usada para examinar a influência da amputação na estabilidade através de um caso de estudo comparando amputados e não amputados para comparação pela medição do centro de pressão (CoP). O estudo usando TI mostrou a incidência de deslocamento do CoP, dando indicação de resposta para manter a estabilidade postural. Este é o primeiro relato de análise de CoP em amputados transitivais usando TI.

No terceiro artigo "Uso de la Tecnología de la Información y Comunicación en educación virtual y su correlación con la Inteligencia Emocional de 
docentes en el Ecuador en contexto COVID-19" da autoria de Zambrano Vacacela Luis Leonardo, propõem uma investigação não experimental, para demonstrar o nível de conhecimento e utilização das Tecnologias da Informação e Comunicação (TIC) na educação virtual e sua correlação com a Inteligência Emocional (IE). Os resultados mostram que há poucas habilidades no uso das TIC e destacam que o aplicativo WhatsApp passou de um meio de comunicação instantânea para uma plataforma de aprendizagem.

De seguida, o quarto artigo "Framework multimodal emocional en el contexto de ambientes dinámicos" da autoria de Ierache Jorge, Sattolo Iris e de Chapperón Gabriela, apresenta os antecedentes do tema da computação afetiva. Aborda a importância dos avanços da tecnologia. São explorados os modelos emocionais dedutivos e categóricos. E são apresentados e discutidos os resultados gerais de um caso de uso realizado.

No quinto artigo "Uso del Estándar ISO/IEC 29110 para Entrenar Estudiantes en Procesos de Ingeniería de Software" da autoria María de León-Sigg , Juan L. Villa-Cisnerose de Blanca E. Solís-Recéndez, propõe-se usar a norma ISO / IEC 29110 para treinar os estudantes universitários a colmatar uma lacuna entre o que é aprendido durante a fase académica e o que realmente precisam de ter conhecimento para trabalhar na indústria de software. Utilizando a abordagem da pesquisa-ação, essa pode ser a solução para auxiliar na eliminação dessa lacuna na sua formação como engenheiros.

O sexto artigo "Estudo Comparativo de Abordagens para Sistemas de Recomendação baseados em Personalidade com uso do serviço IBM Watson Personality Insights" da autoria de Janderson Jason B. Aguiar, Joseana M. F. R. de Araújo e de Evandro de B. Costa , foi analisado se, com os avanços na detecção da personalidade, as abordagens de Filtragem Colaborativa baseadas em personalidade continuam a melhorar a acurácia das abordagens. No caso de uso, foram considerados utilizadores/clientes com características de personalidade inferidas via IBM Watson Personality Insights. Os resultados indicaram a possibilidade de melhorar a acurácia ao empregar outras abordagens.

No sétimo artigo "Estudo da imagem da região turística de Lisboa" da autoria João H. da Luz Cerdeira e Adriana Fernandes ilustra que para a reinvenção e reforma do setor do turismo se terá de investir fortemente na criação e gestão da perceção dos turistas sobre a imagem dos destinos turísticos, (prejudicado fortemente desde março de 2020, quando foi fortemente afetada pela pandemia Covid-19). O objetivo deste pretende analisar a imagem da região de Lisboa através da utilização de uma aplicação que utiliza uma metodologia de análise de três componentes de interações do turista com o destino: imagem Designativa, Estimativa e Prescritiva. Os resultados apresentados revelam a caracterização da região de Lisboa.

O oitavo artigo "Garantia de Privacidade Versus Utilidade dos Dados em Anonimização: um estudo no ensino superior" da autoria de Paula Prata, Maria Eugénia Ferrão, Wilson Santos e de Gonçalo Sousa, ilustra um processo de anonimização, comparando para vários modelos de privacidade a perda de informação e a utilidade do conjunto de dados resultante. Encontrar o equilíbrio entre privacidade e utilidade é um 
desafio que pode ser mais facilmente alcançado por quem melhor conhece o significado dos dados e dos objetivos que se pretendem alcançar com eles.

Por fim, no último e nono artigo "Previsões de mortalidade e de esperança de vida mediante combinação Bayesiana de modelos: Uma aplicação à população portuguesa" da autoria de Jorge M. Bravo e de Mercedes Ayuso, desenvolve-se um modelo de previsão da mortalidade específica por idade e da esperança de vida mediante combinação Bayesiana de modelos (Bayesian Model Ensemble). Os modelos são calibrados à população portuguesa desagregada por sexo, tendo por base informação estatística relativa ao período $1960-2018$ e às idades no intervalo 60-125 anos. São efetuadas previsões até 2050. Os resultados obtidos antecipam a continuação do aumento da esperança de vida em Portugal.

\section{Agradecimentos}

Os editores gostariam de finalizar agradecendo a todos os que de forma direta ou indireta colaboraram com sucesso na produção deste número, incluindo os autores, comissão científica e equipa editorial, entre muitos outros. Através do seu interesse, participação e da qualidade e rigor do seu trabalho científico, agora publicado na RISTI, esperamos que continue a ser promovida a expansão da investigação qualitativa e quantitativa numa área tão relevante como é a dos Sistemas e Tecnologias de Informação.

\section{Referências}

Almeida, C. P., \& Araújo, R. C. S. (2019). Gestão da Inovação em um Ambiente Educacional - Aplicação da Metodologia ITIL, Revista Académica do curso de administração, 1(1), 1-9.

Andrade, A. M., Langhi, C., \& Okano, M. T. (2019). Gestão do Conhecimento e Sistemas de Informação em Autarquia Pública do Estado de São Paulo. South American Development Society Journal, 5(14) 222. http://dx.doi.org/10.24325/issn.24465763.v5i14p222-235.

Araújo, D., Lima, D., Campos, P., Azevedo, V., \& Barbosa, J. (2019). Como as Tecnologias de Informação e Comunicação Podem Revolucionar a Saúde e a Medicina. Revista Científica E-Locução, 1(15), 23. http://periodicos.faex.edu.br/index.php/eLocucao/article/view/187

Civi, E. (2000). Knowledge management as a competitive asset: a review. Market. Intell. Plann., ,18(4), 166-174. https://tinyurl.com/yajozlkr. Acesso em: 27 dez. 2020.

Hummel, G. S. (2006). eHealth- O iluminismo digital chega à saúde. As tecnologias de informação e comunicação revolucionando o setor de saúde. São Paulo: Editora STS.

Marcondes, J. S., (2019) https://gestaodesegurancaprivada.com.br/sistema-deinformacao-o-que-e-conceitos/. Acesso em: 27 dez. 2020. 
Ribeiro, J. S A. N. (2019). Gestão do conhecimento e sistemas de informação na cadeia de suprimentos global. Revista Brasileira de Biblioteconomia e Documentação (São Paulo), 15(2), 251-289. https://rbbd.febab.org.br/rbbd/article/view/1203. Acesso em: 27 dez. 2020.

Rivas, F. (1989). Estruturas Organizativas e Informação na Empresa. Lisboa: Domingos Barreira.

Turban, E., McLean, E., \& Wetherbe, J. (1999). Information Technology for Management - Making Connections for Strategic Advantage. New York: John Wiley. 